\title{
Scheduling of QoS Guaranteed Services in DS-CDMA Uplink Networks: Modified Weighted G-Fair Algorithm
}

\author{
Young Min Ki, Eun Sun Kim, and Dong Ku Kim \\ Yonsei University, Dept. of Electrical and Electronic Engineering \\ 134 Shinchon Dong, Seodaemun Gu, Seoul, Korea \\ \{mellow, esunkim, dkkim\}@yonsei.ac.kr \\ http://mcl.yonsei.ac.kr
}

\begin{abstract}
In this paper, the modified weighted g-fair (MWGF) scheduling scheme with rise over thermal (RoT) filling is proposed for QoS guaranteed services in DS-CDMA uplink networks, in which RoT is directly related to the loading factor of a wireless network. The proposed scheme computes priorities of QoS guaranteed traffic for each slot based upon the weighted values of the QoS factor, channel factor, and fairness factor. Once priorities are assigned, power and rate used for each user are computed within a limited RoT by using RoT filling, which adjusts the transmit rate of the traffic assigned least priority. The proposed algorithm increases more throughput by 6 to $10 \%$ compared to that of the current autonomous rate control (ARC) scheme. It also lowers transmission delays and ensures more fairness in delay outages than the round robin scheduler and ARC scheme.
\end{abstract}

\section{Introduction}

In $3 \mathrm{G}$ wireless networks, link adaptation and channel scheduling are key techniques used for dynamic resource management. In a cdma2000 1xEV-DO type downlink, adaptive modulation and coding (AMC) as well as proportional fairness $(\mathrm{PF})$ scheduling have improved the overall performance of high data rate services [1]. The modified largest weight delay first (MLWDF) [2] and exponential (EXP) [3] algorithms have been proposed for real-time services. An adaptive $\mathrm{EXP} / \mathrm{PF}$ algorithm has also been proposed for applications in multiple QoS service traffic experienced in a 1xEX-DO downlink [4].

The proposed uplink system should offer QoS to multimedia traffic as well as control multiple access interference, as not to overload the system. A cdma2000 1x (IS-2000) like uplink control scheme called autonomous data rate control (ARC) is employed in 1xEV uplink [5]. This distributed control scheme can neither support the various QoS requirements nor hold RoT constraint, which is associated with system loading, and used in highly traffic loaded environments. The weighted proportional fairness (WPF) algorithm was proposed to schedule the best effort services for the uplink [6]. However, it did not yet consider delay 
requirements necessary for efficient QoS guaranteed services. In this paper, we take into consideration a scheduling algorithm for applications in QoS guaranteed services in cdma2000 type uplink, and propose a modified weighted g-fair (MWGF) algorithm for by using the notion of the WPF [6], MLWDF [2] and $\mathrm{G}$-fair [7]. The total performance of the proposed algorithm is evaluated with simulations and compared with those of the ARC scheme as well as round robin scheduling algorithm.

The remainder of the paper is organized as follows. In section 2 , the conventional uplink MAC algorithm for cdma2000 is presented. In section 3, proposed uplink scheduling schemes are presented. The total performance of the schemes is studied in section 4 by simulation. Finally, conclusions are made in Section 5 .

\section{Uplink MAC Algorithm for CDMA Cellular Networks}

The uplink MAC is used to control the data rate at which the access terminals transmit. The access networks control the data rates of the mobile users by using two mechanisms: Reserve rate limit, and Reverse activity bit (RAB) and the transition probabilities.

\subsection{Reverse Rate Limit}

In autonomous data rate control (ARC) scheme, the user can send data anytime regardless of transmitting of the uplink traffic channel request message. The user initially starts to send his or her message at the lowest data rate. Then, if the user receives an idle RAB from the base station (BS), it will increase the data rate to the next higher level or transmission power level. If the user receives a busy RAB, it will decrease the data rate to the next lower level or transmission power level. However, when the data rate reaches its minimum, maximum, or the required transmit power of a mobile user exceeds the maximum available transmit power, the current data rate is maintained.

\subsection{Reverse Activity Bit and Transition Probabilities}

The RoT, defined as the total received power to thermal noise ratio occurring in a specific BS, is the parameter used to determine the network's capacity. The BS measures the received RoT given in (1).

$$
R o T=\frac{\sum_{i}^{\text {active in cell }} P_{T X, i} L_{i}+\sum_{k}^{\text {other cells }} I_{k}+N_{0} W}{N_{0} W}
$$

where $P_{T X, i}$ is the transmit power of the $i$-th user, $L_{i}$ is the radio path loss, $I_{k}$ is the other cell interference from the $k$-th cell, and $N_{0} W$ is the thermal noise. The RAB is determined by equation (2) and broadcast to all users in the cell, in a regular manner.

$$
R A B= \begin{cases}\text { idle, } & \text { if } R o T<R o T_{\text {setpoint }} \\ \text { busy, } & \text { if } R o T>R o T_{\text {setpoint }}\end{cases}
$$


where $R o T_{\text {setpoint }}$ is the threshold of the RAB operation. This threshold is generally set smaller than $R o T_{\text {limit }}$ which is the pre-determined receive interference level of BS. Fig. 1 shows the autonomous data rate transition process along with transition probabilities. Two rate transition probabilities can be defined for use in rate transition. If the $\mathrm{RAB}$ is idle, the user can transit to the higher data rate with the probability, $p$ as shown in Fig. 1 (a). If the RAB is busy, the user can transit to the lower data rate with the probability, $q$ as presented in Fig. 1 (b).

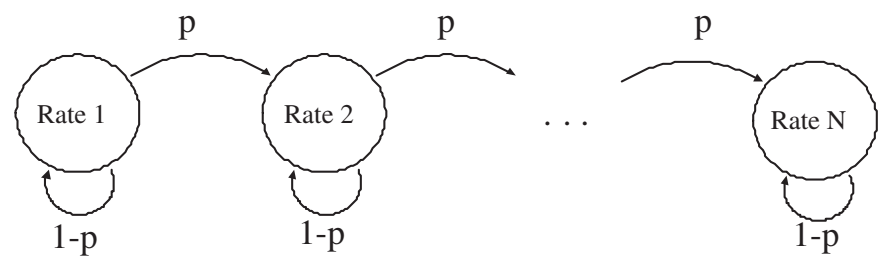

(a) Rate transition when RAB is idle

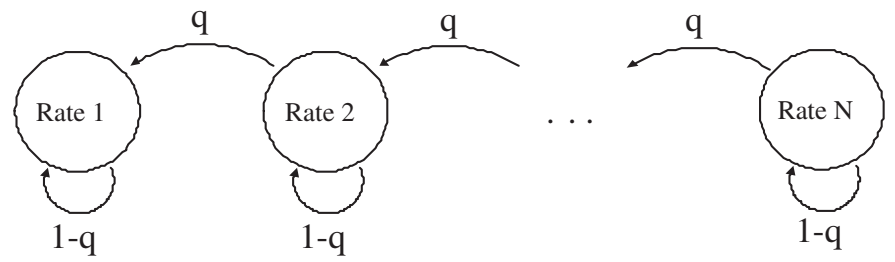

(b) Rate transition when RAB is busy

Fig. 1. Reverse rate transition process (N: the number of available data rates)

\section{Uplink Scheduling for CDMA Cellular Networks}

In a DS-CDMA uplink, there are two system constraints. One is the limited transmit power of each user and the other is the receive interference level of the base station (BS). Therefore, the uplink scheduler is used to determine how to allocate the available amount of power to each user without exceeding the predetermined interference level as well as priority determination [6][8]. The channel scheduler of a cdma2000 uplink can serve some users simultaneously within the pre-determined RoT limit value, while the channel scheduler of a TDM type $1 \mathrm{xEV}$-DO downlink allocates the full power of the BS to one user in each slot.

Since the BS does not identify queue status of each user's traffic, the scheduling and rate/power allocation should be determined at the BS by data rate requests and the queue status from users. Then, the uplink scheduling scheme has three stages as shown in Fig. 2. First, each user requests a desired transmit data rate and informs the queue status to BS. Secondly, the BS performs the 
scheduling process by executing two phases, i.e. scheduling and rate/power allocation. Since the cdma2000 uplink has two constrains on the available amount of transmit power as well as receive interference, which make joint determination of scheduling and simultaneous rate/power allocation very difficult, scheduling is first processed and subsequently followed by power/rate allocation. After scheduling and rate/power allocation processes are completed, the users can transmit their traffic by using contention-free dedicated resources. Since it required three stages to transmit data from initial demand of channel, uplink scheduling has at least two potential delay slots.

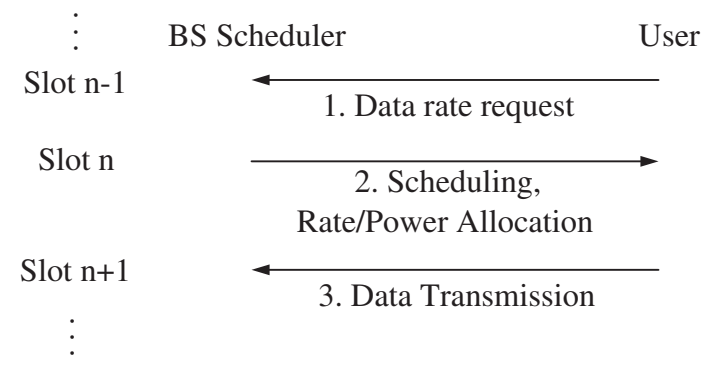

Fig. 2. Uplink scheduling schemes for a DS-CDMA system

Most distributed control schemes can neither support various QoS requirements nor hold the RoT constraint which is associated with system loading, and designed for use in highly traffic-loaded environments. The WPF centralized scheduling scheme was proposed to schedule the best effort services for use in the uplink [6]. We take into consideration a scheduling algorithm for use with QoS guaranteed services, and propose innovative MWGF algorithms.

\subsection{Weighted Proportional Fairness (WPF)}

In [6], the WPF algorithm was proposed for the uplink in order to ensure best effort services and results demonstrated the trade-off between throughput and fairness. The scheduler determines user priorities by applying the following metrics:

$$
P_{i}=w_{i} \cdot\left(\frac{R_{i}^{r e q}}{R_{i}}\right)^{1 / \alpha}
$$

where $w_{i}$ can be corrected to existing channel conditions in order to give a higher priority to the mobile users with better channels. Hence, it introduces another degree of freedom in adjusting fairness. The expression $R_{i}^{r e q} / R_{i}$ is a well-known PF factor [6]. 


\subsection{Proposed Scheduling Algorithm: Modified Weighted G-Fair (MWGF)}

We adopt the MWPF scheduling algorithm for used in QoS guaranteed services, whose priority metrics are given in (4):

$$
P_{i}=C_{i} \cdot\left(Q_{i}\right)^{a} \cdot\left(W_{i}\right)^{b} \cdot\left(F_{i}\right)^{c}
$$

where $C_{i}$ is the service grade factor of the $i$-th user and can be determined by the user's subscribing class grade such as gold, silver or bronze. The symbol $Q_{i}$ represents the QoS factor and is given as (5):

$$
Q_{i}=\frac{D_{i}}{D_{i}^{r e q}}
$$

where $D_{i}$ is the head of line (HOL) delay and $D_{i}^{r e q}$ is the required delay QoS class in which the $i$-th user belongs. Normalization factor identified by $D_{i}^{r e q}$ can have a priority value weighted by the amount of each user's delay sensitivity. In [6], the forward link signal to interference and noise ratio (FL SINR) was used as a channel factor. However, we assume that the uplink pilot channel in a cdma2000 1xEV provides continuous channel estimation, so that the channel factor could be set to the uplink path loss value given in (6), instead of FL SINR:

$$
W_{i}=\mathrm{UL} \text { Path } \operatorname{Loss}_{i}
$$

We also consider the normalized uplink path loss presented in (7), as another candidate of the channel factor:

$$
W_{i}=\frac{\text { UL Path } \operatorname{Loss}_{i}}{\text { UL Average Path Loss } i}
$$

Finally, $F_{i}$ is the fairness factor that can be set to the G-Fair factor given in (8).

$$
F_{i}=\frac{R_{i}^{r e q}}{R_{i}} \cdot \frac{h_{i}\left(\overline{R_{i}^{r e q}}\right)}{\overline{R_{i}^{r e q}}}
$$

where $R_{i}^{r e q} / R_{i}$ is a well-known $\mathrm{PF}$ factor. The uplink rate request $R_{i}^{r e q}$ is determined by the user's buffer status and does not include the channel condition as defined in (9).

$$
R_{i}^{r e q}=\min (\text { traffic rate, regional rate, service grade rate) }
$$

where traffic rate represents the data rate required to transmit the remaining data volume. When the regional rate is the user's maximum available transmission rate predetermined by user's distance from the BS, and the service grade rate is the maximum available transmission rate associated with the user's service grade [5]. The symbol $h(x)$ represents a user-specific function that specifies the fairness behavior. If $h(x)$ is constant, the scheduler attempts to provide the same average throughput to all users. If $h(x)=x$, the g-fair scheduler becomes the proportional fair scheduler that we currently use [7]. 


\subsection{Proposed Rise over Thermal Filling}

Since cdma2000 uplink has two constrains concerning the limited transmit power and receive interference, which make joint determination of scheduling and simultaneous power/rate allocation very difficult. Therefore, scheduling is first processed and subsequently followed by power/rate allocation. Once user requests are prioritized, then the transmit power, $P_{T X, i}$ is calculated at the BS as follows,

$$
\left(\frac{E_{b}}{N_{0}}\right)_{i, r e q}=\frac{W_{i}}{R_{i}^{r e q}} \frac{P_{T X, i} L_{i}}{I_{\text {total }}},
$$

where $E_{b} / N_{0}$ is the bit energy per noise ratio, $W$ is the signal bandwidth, $L$ is the channel path loss, and $I_{\text {total }}$ is the total interference [9]. Once the required power levels are calculated, the scheduler starts allocating power to users in a decent order of priorities while computing the RoT value given in (1), and the BS can only allocate power levels within the available RoT limit value. This RoT filling technique is applied to the scheduling schemes. If the RoT value exceeds the RoT limit, while computing RoT, the data rate of the last filled user is decreased to the nearest level so that the computed RoT value does not exceed the RoT limit. Then, all users start transmission with these given rates and powers.

\section{Simulations: QoS Guaranteed Service Assumption}

\subsection{Simulation Environments}

Six video telephone users are assumed to be uniformly distributed within a cell. They have the minimum required rate of $64.3 \mathrm{kbps}$ and very strict slot delay constraints (around 10 to $30 \mathrm{msec}$ ). These 19 circular cells with a $1.44 \mathrm{~km}$ cell radius are assumed, but simulation results have been extracted from the center cell only. The uplink reuse fraction is set at 0.55 . Other simulation parameters are listed as follows:

- Signal BW: $1.2288 \mathrm{MHz}$

- Target Eb/No : $3.10 \mathrm{~dB}$ (for all users)

- Tx power limit: $23.0 \mathrm{dBm}(200 \mathrm{~mW})$

- Slot size: 1.67 msec (1xEV-DO)

- Propagation model: $28.6+35 \log _{10}\left(d_{\text {meter }}\right) \mathrm{dB}$

- Shadowing: Log normal with a standard deviation of $8.9 \mathrm{~dB}$

- Rayleigh fading with Doppler frequency, $10.0 \mathrm{~Hz}$

- Max propagation loss: $146.0 \mathrm{~dB}$

- BS antenna gain: $5.0 \mathrm{~dB}$

- Thermal noise density: $-174.0 \mathrm{dBm} / \mathrm{Hz}$

- RoT limit: $10.0 \mathrm{~dB}$

The available transmission data rate of users can be selected according to 
Table 1. User's average geometry and regional rate

\begin{tabular}{ccc}
\hline User & Average distance from BS $[\mathrm{m}]$ & Regional rate $[\mathrm{kbps}]$ \\
\hline 1 & 72.01 & 1,024 \\
2 & 996.86 & 614.4 \\
3 & 724.11 & 1,024 \\
4 & 129.58 & 1,024 \\
5 & 903.46 & 614.4 \\
6 & 541.41 & 1,024 \\
\hline
\end{tabular}

Table 2. Investigated MWGF algorithms applied in various simulation scenarios

\begin{tabular}{lccc}
\hline Factor & Algorithm 1 & Algorithm 2 & Algorithm 3 \\
\hline 1. Service grade & No use & No use & No use \\
2. QoS & Normalized delay & Normalized delay & Normalized delay \\
3. Channel & UL path loss & Normalized path loss & Normalized path loss \\
4. Fairness & Conventional PF & Conventional PF & G-Fair $(h(x)=1)$ \\
\hline
\end{tabular}

1xEV data rates: 0(NULL), 9.6, 19.2, 38.4, 76.8, 153.6, 307.2, 614.4, and 1,024 kbps [5]. The regional rate differentiation is also applied to our simulation. The distances from the BS to the user as well as regional rate constraints of users are shown as Table 1 . We have investigated three schemes: ARC, MRR, and the MWGF scheduler:

(Scheme 1) ARC scheme: simulations were conducted for the ARC of $9.0 \mathrm{~dB}$ RoT set point and $8.0 \mathrm{~dB}$ RoT set point respectively. The rate transition probabilities were set to $p=q=0.5$.

(Scheme 2) Modified round robin (MRR) scheduler: a priority rotation method is used, in which the priorities were rotated among users. For example, if the priority orders were (User 1, User 2, User 3, User 4, User 5, User 6) in the $n$-th slot, the priority orders were (User 2, User 3, User 4, User 5, User 6, User 1 ) in $(n+1)$-th slot, and the priority orders will be (User 3, User 4, User 5, User 6 , User 1, User 2) in the next slot. The priorities for the subsequent slots will be rotated in every slot.

(Scheme 3) Proposed MWGF scheduler: the proposed scheduling algorithms were investigated for three different factor choices. The discrepancy calculated three versions is shown in Table 2. The weighting indexes are set to $a=2.0 \sim 6.0, b=0.5, c=1.0$. These values were chosen through trials, experimentations and simulations in order to find nearly optimal values.

\subsection{Throughput and Delay Performance}

Fig. 3 shows the total throughput of the investigated schemes. The scheduling schemes, i.e. MRR and MWGF, produce more throughput when compared to 
those of the ARC schemes by 6 to $10 \%$. These improvements originate from the multi-user diversity gain of the centralized scheduling schemes [1]. Fig. 4 shows the user throughput of the best geometry user (User 1) and that of the worst geometry user (User 2). Since User 2 has excessively poor channel conditions, User 2 can not be served with the $64.3 \mathrm{kbps}$ minimal rate used in ARC schemes. Therefore, User 2 needs to be allocated more channel resources.

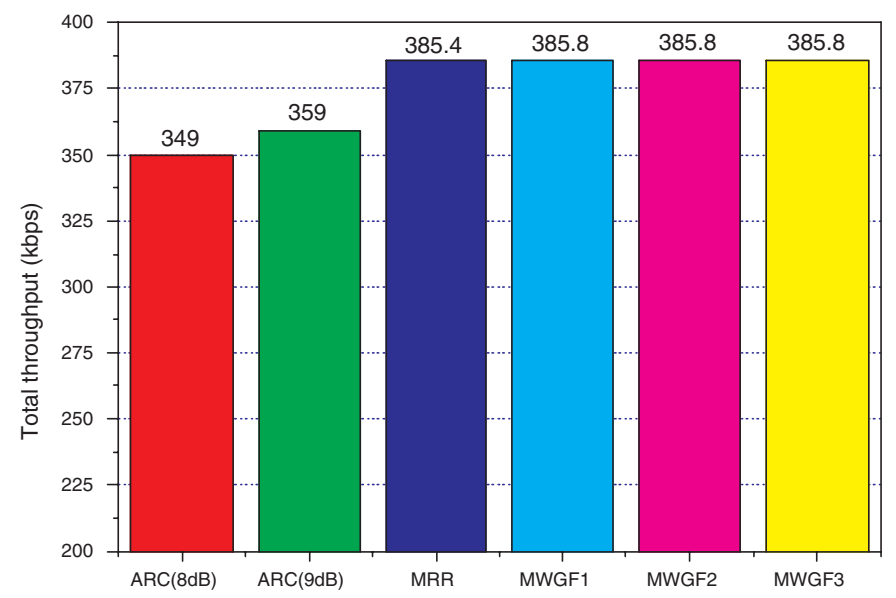

Fig. 3. Total throughput for the investigated schemes

Table 3 shows the average transmission delay of the best geometry user (User 1) and that of the worst geometry user (User 2). In the MRR scheme, it is shown that throughput of User 2 can be served with a transmission speed around the minimal rate, but User 2 suffers very a large delay compared to the MWGF scheme. This indicates that the round robin can allocate channel resources to users by fairly with reference time, but cannot allocate the channel resources in an adequate channel scheduling time.

\subsection{Delay Outage Probability Performance}

Fig. 5 displays the delay outage probability of users in the MRR algorithm. It is shown that user 2 suffers a very large delay outage probability. Fig. 6, 7 and 8 show the outage delay probability of the proposed algorithms: Algorithm 1, Algorithm 2, and Algorithm 3. In Algorithm 2 and Algorithm 3, the difference between user with the best delay performance and the worst one is smaller than in Algorithm 1. The figures indicate that the $10^{-2}$ outage of Algorithm 1 can be achieved within around $35 \mathrm{msec}$, and those of Algorithm 2 and Algorithm 3 can be obtained within 20 msec. Algorithm 2 and Algorithm 3 produce increased 


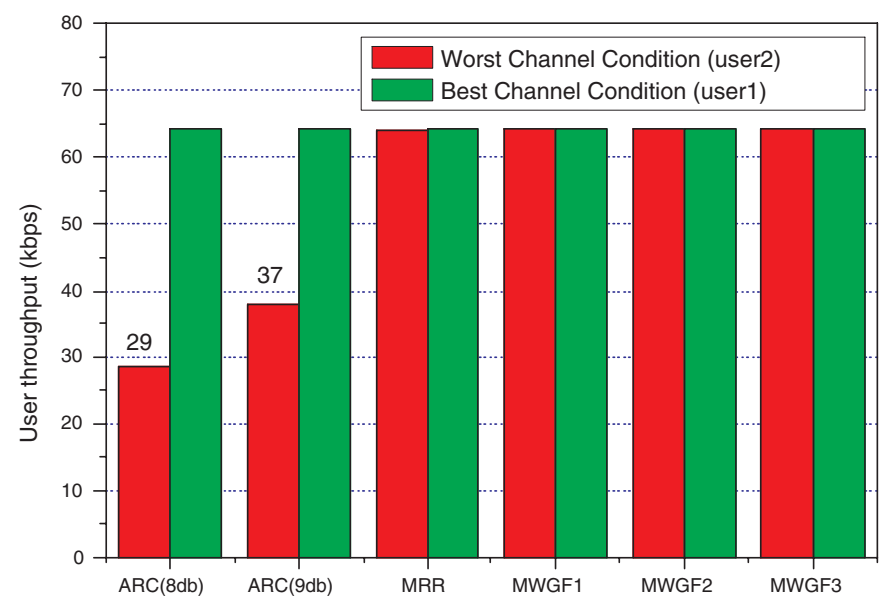

Fig. 4. Throughput for the best geometry user and the worst geometry user

Table 3. Average delays for the best geometry user and the worst geometry user

\begin{tabular}{lcc}
\hline & $\begin{array}{c}\text { Best channel condition } \\
\text { (User 1) }\end{array}$ & $\begin{array}{c}\text { Worst channel condition } \\
\text { (User 2) }\end{array}$ \\
\hline ARC (8 dB) & $45.94 \mathrm{msec}$ & $464.0 \mathrm{sec}$ \\
ARC (9 dB) & $30.27 \mathrm{msec}$ & $376.0 \mathrm{sec}$ \\
MRR & $7.03 \mathrm{msec}$ & $4.53 \mathrm{sec}$ \\
MWGF 1 & $9.03 \mathrm{msec}$ & $16.88 \mathrm{msec}$ \\
MWGF 2 & $10.15 \mathrm{msec}$ & $9.93 \mathrm{msec}$ \\
MWGF 3 & $10.32 \mathrm{msec}$ & $9.59 \mathrm{msec}$ \\
\hline
\end{tabular}

fairness when compared to Algorithm 1 and the MRR with regard to delay outage.

\subsection{Effect of Weighting Index on MWGF Scheduling Scheme}

Fig. 9 shows the average transmission delay of the proposed schemes according to their weighting indexes. In this figure, we set the QoS weighting index $a$ to 2.0 to 6.0 . Symbols $b$ and $c$ are fixed at $(b=0.5, c=1.0)$. Therefore, as QoS weight a increases, average transmission delays decrease. Since the channel factor of Algorithm 1 is not normalized, the effect of the increase of a is the largest among the proposed Algorithms. Fig. 10 shows average received RoT level of the proposed schemes, calculated according to their weighting indexes. It is shown that as a increases, the RoT level of Algorithm 1 decreases from 8.6 $\mathrm{dB}$ to $8.3 \mathrm{~dB}$, and those of Algorithm 2 and Algorithm 3 increase $8.25 \mathrm{~dB}$ to 8.4 $\mathrm{dB}$ respectively. 


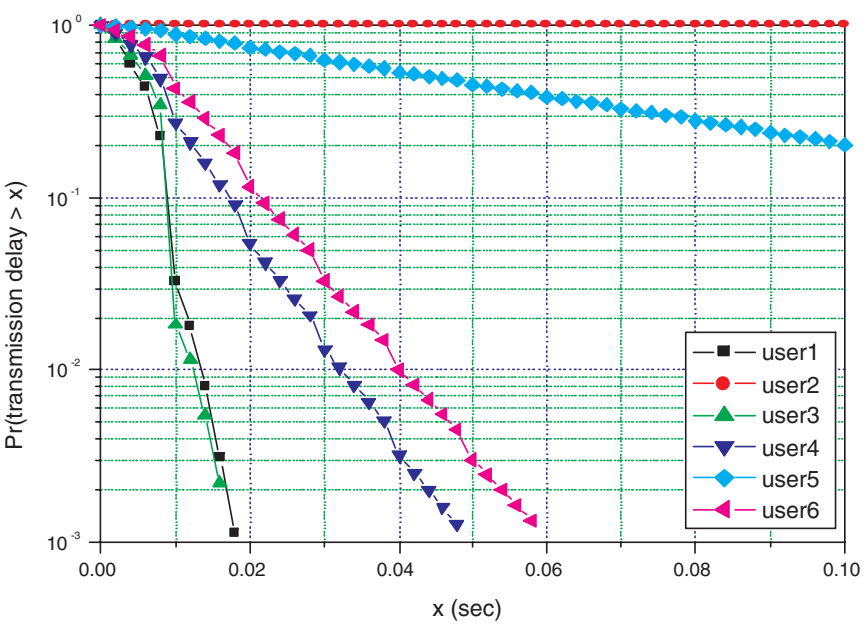

Fig. 5. Delay outage probability of the modified round robin scheduling scheme

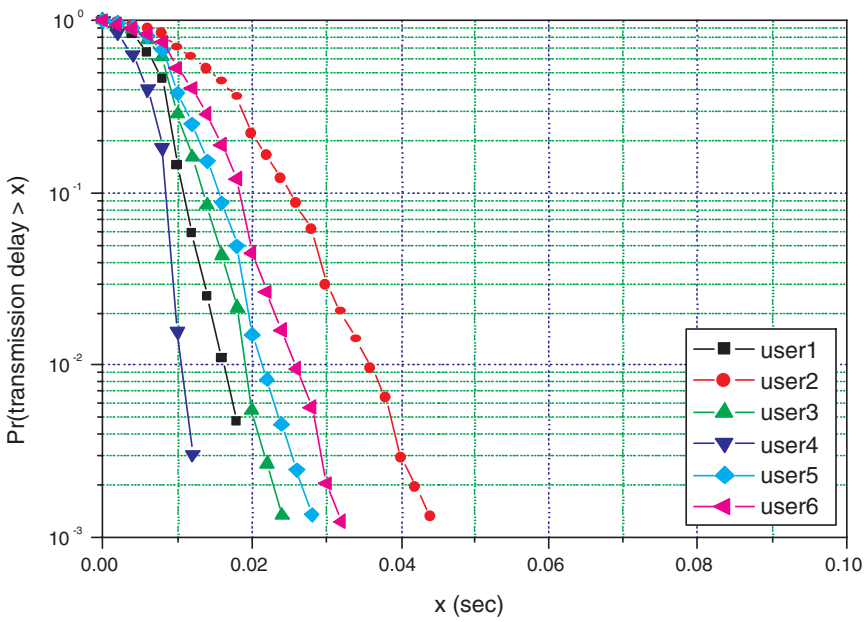

Fig. 6. Delay outage probability of the proposed MWGF Algorithm 1 (weighting index: $a=4.0, b=0.5, c=1.0)$ 


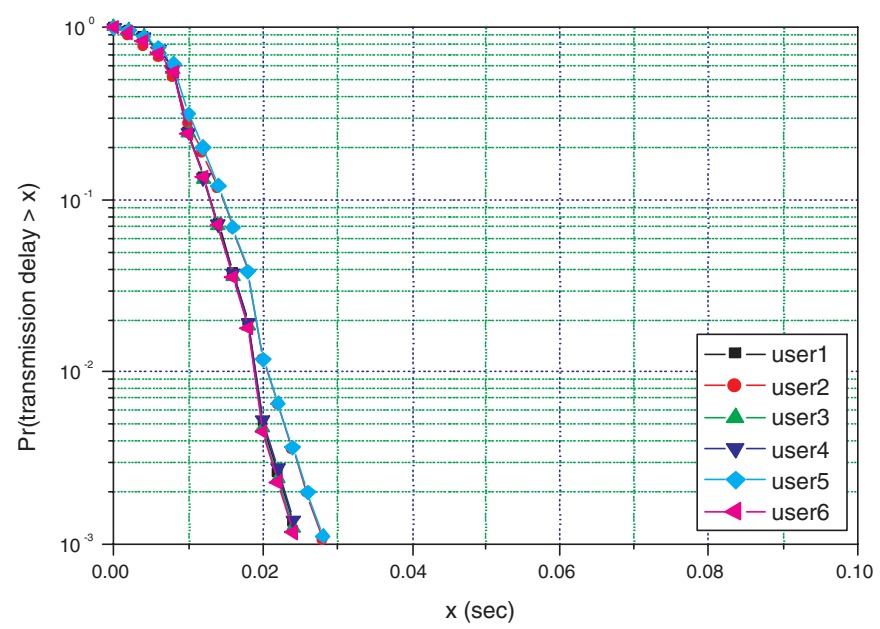

Fig. 7. Delay outage probability of the proposed MWGF Algorithm 2 (weighting index: $a=4.0, b=0.5, c=1.0$ )

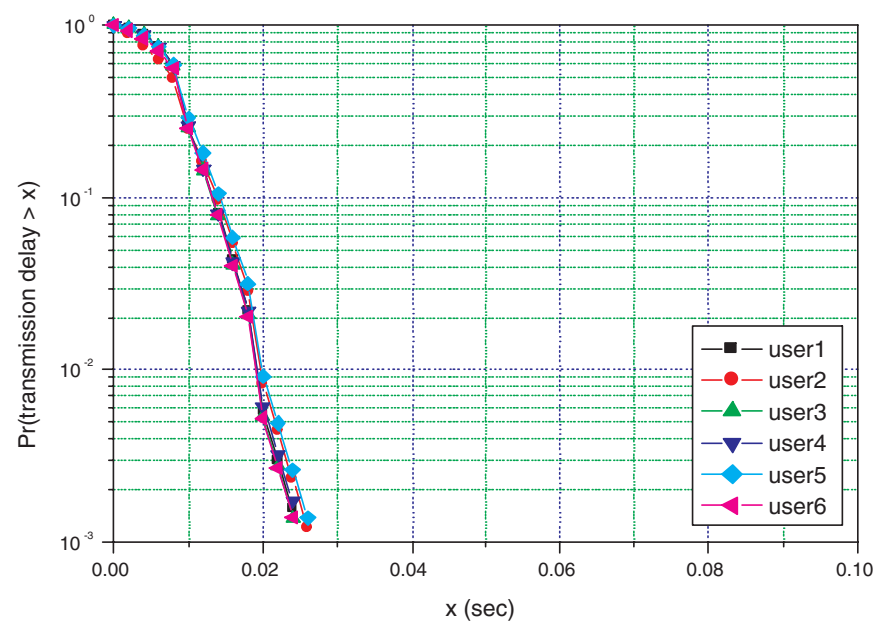

Fig. 8. Delay outage probability of the proposed MWGF Algorithm 3 (weighting index: $a=4.0, b=0.5, c=1.0$ ) 


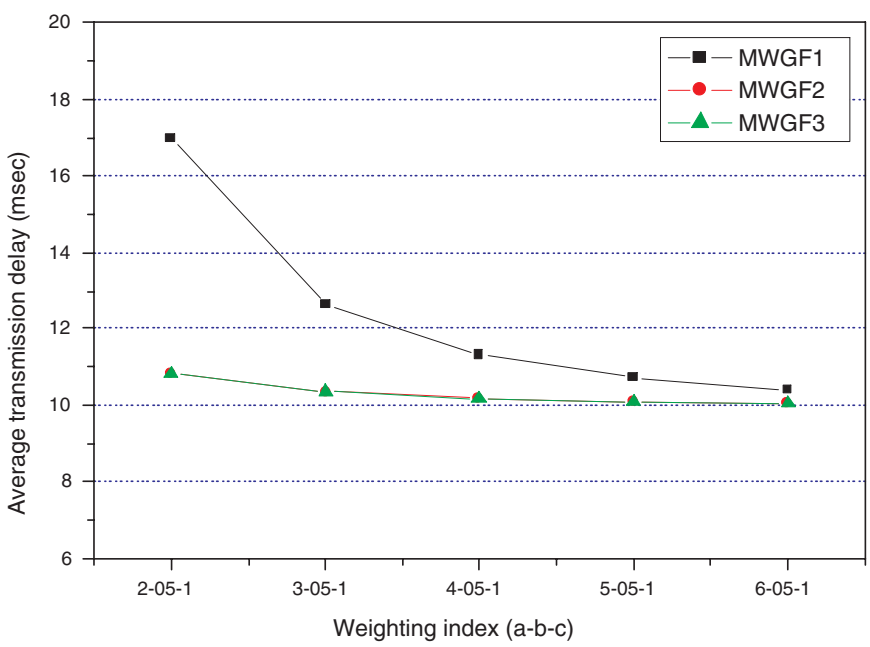

Fig. 9. Average transmission delay of the proposed schemes versus weighting indexes (weighting index: $a=2.0 \sim 6.0, b=0.5, c=1.0$ )

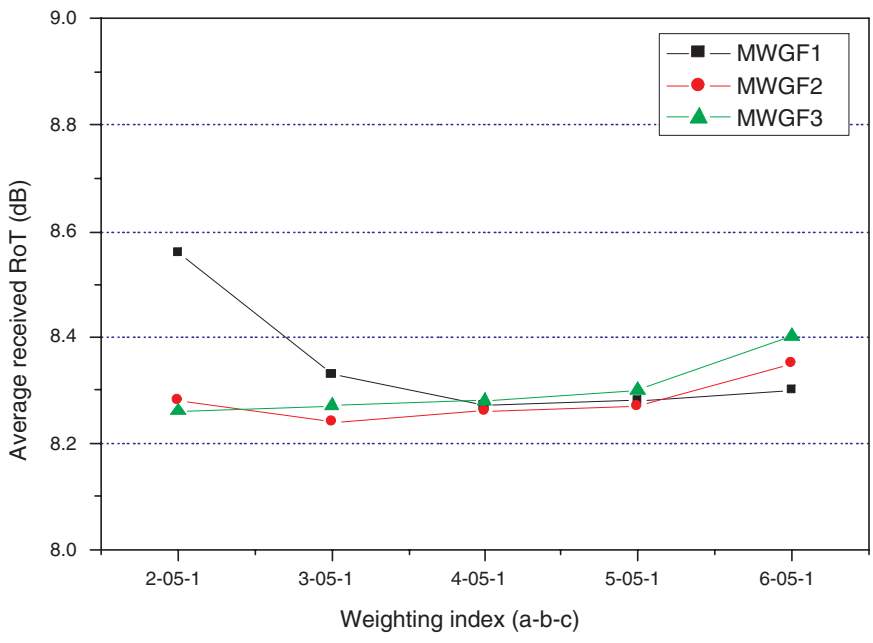

Fig. 10. Average received RoT level of the proposed schemes versus weighting indexes (weighting index: $a=2.0 \sim 6.0, b=0.5, c=1.0$ ) 
The proposed algorithm produces increased throughput of 6 to $10 \%$, when compared with the autonomous rate control and demonstrates lower transmission delays as well as increased fairness than the round robin scheduler. It also displays a 8 to $8.5 \mathrm{~dB}$ received RoT level.

\section{$5 \quad$ Concluding Remarks}

The MWGF scheduling scheme was proposed for use in QoS guaranteed services in a cdma2000 type uplink. The proposed scheme mainly focuses on computing priorities used for QoS guaranteed traffic based on the weighted values of QoS, channel, and fairness factor. Once priorities were assigned, power and rate for each user were computed within a limited RoT level by using RoT filling, which adjusted the overall user traffic transmit rate. The proposed algorithm produces 6 to $10 \%$ increased throughput when compared to that of autonomous rate control (ARC) scheme and ensures lower transmission delays as well as more fairness in delay outages than either the round robin scheduler or the ARC scheme. It is also demonstrated that the choice of weighting index by the proposed scheduling algorithm allows trading off received RoT level and transmission delays.

Acknowledgment. This work was supported by grant No. (R01-2002-00000531-0) from the Basic Research Program of the Korea Science \& Engineering Foundation. Also, the work was supported by Qualcomm Incorporated through Qualcomm Yonsei CDMA Research Center. The authors thank Jelena Damnjanovic, Jack Holtzman and Edward Tiedemann for valuable discussions and comments.

\section{References}

1. Bender P., Black P., Grob M., Padovani M., Sindhushayana N., Viterbi A.: CDMA/HDR: A Bandwidth-Efficient High-Speed Wireless Data Service for Nomadic Users. Communications Magazine, Vol. 38. IEEE. (2000) 70-77

2. Andrews M., Kumaran K., Ramanan K., Stolyar A., Whiting P., Vijayakumar R,: Providing quality of service over a shared wireless link. Communications Magazine, Vol. 39. IEEE. (2001) 150-154

3. Shakkottai S., Stolyar A.: A study of Scheduling Algorithms for a Mixture of Realand Non-Real-Time Data in HDR. 17th International Teletraffic Congress (ITC17), (2001)

4. Rhee J., Kim D.: Scheduling of Real/Non-real Time Services in an AMC/TDM Systems: EXP/PF Algorithm. Lecture Notes in Computer Science, Vol. 2524. SpringerVerlag, Berlin Heidelberg New York (2003) 506-513

5. Koo C., Bae B., Jung J.: An Efficient Reverse Link Control Scheme for 3G Mobile Communication Systems. IST Mobile \& Wireless Telecommunications Summit (2002)

6. Damnjanovic J., Jain A., Chen T., Sarkar S.: Scheduling the cdma2000 Reverse Link. IEEE 56th Vehicular Technology Conference (2002) 
7. Application Note: G-fair Scheduler. 80-H0551-1 Rev. B. Qualcomm Incorporated. (2002)

8. Padovani R.: The Application of Spread Spectrum to PCS has Become Reality: Reverse Link Performance of IS-95 Based Cellular Systems. Personal Communications, Vol. 1, IEEE. (1994) 28-34

9. Dimou K., Godlewski P.: MAC Scheduling for Uplink Transmission in UMTS WCDMA. IEEE 54th Vehicular Technology Conference (2001)

10. Application Note: PLMAC Algorithm for IS-856 (1xEV). CL93-V3762-1 X1. Qualcomm Incorporated. (2001)

11. Shakkottai S., Rappaport T. S.: Cross-Layer Design for Wireless Networks. Communications Magazine, Vol. 41. IEEE. (2003) 74-80

12. TIA/EIA/IS-856. cdma2000 High Rate Packet Data Air Interface Specification, (2001)

13. cdma2000 release C. C.S000X-C, (2002)

14. 3GPP release 5. TS-25.211-25.214, (2002)

15. S. Keshav: An Engineering Approach to Computer Networking: ATM Networks, the Internet, and the Telephone Network. Addison Wesley. (1997)

16. Recommendation ITU-R M.1225, Guideline for Evaluation of Radio Transmission Technologies for IMT-2000, (1997) 